\title{
CHECK-LIST OF THE PIPERACEAE OF EQUATORIAL GUINEA
}

\author{
by \\ MAXIMILIANO FERO', FRANCISCO CABEZAS ${ }^{2}$, CARLOS AEDO $^{2}$ \& MAURICIO VELAYOS ${ }^{2}$ \\ ' Escuela Universitaria de Estudios Agropecuarios, Pesca y Forestal \\ de la Universidad Nacional de Guinea Ecuatorial. Malabo \\ <sogosote@yahoo.es> \\ ${ }^{2}$ Real Jardín Botánico, CSIC. Plaza de Murillo, 2. E-28014 Madrid \\ <cabezas@ma-rjb.csic.es><aedo@ma-rjb.csic.es><velayos@ma-rjb.csic.es>
}

\begin{abstract}
Resumen
Fero, M., F. Cabezas, C. Aedo \& M. Velayos (2003). Catálogo de las Piperaceae de Guinea Ecuatorial. Anales Jard. Bot. Madrid 60(1): 45-50 (en inglés).

Se presenta el catálogo florístico de la familia Piperaceae para Guinea Ecuatorial, en el que se recogen un total de 13 especies englobadas en tres géneros. En Annobón están presentes tres especies, 13 en Bioko y cinco en Río Muni. Peperomia es el género mejor representado, con 10 especies. Los otros dos géneros, Pothomorphe y Piper, están representados por una y dos especies, respectivamente. Se han recopilado y revisado las referencias bibliográficas previas para la zona hasta un total de 223 citas. Peperomia molleri y Piper capense se citan por primera vez para Río Muni.
\end{abstract}

Palabras clave: Piperaceae, Guinea Ecuatorial.

\begin{abstract}
Fero, M., F. Cabezas, C. Aedo \& M. Velayos (2003). Check-list of the Piperaceae of Equatorial Guinea. Anales Jard. Bot. Madrid 60(1): 45-50.

A check-list of the Piperaceae from Equatorial Guinea is presented, with 13 species. Three species are known from Annobon, 13 species from Bioko and 5 species from Río Muni. The best represented genus is Peperomia, with 10 species. The other two genera, Piper and Pothomorphe, are represented by two and one species. respectively. Additionally, bibliographic references on Piperaceae of Equatorial Guinea (223 records) have been gathered and revised. Peperomia molleri and Piper capense are recorded as new to Río Muni.
\end{abstract}

Key words: Piperaceae, Equatorial Guinea.

\section{INTRODUCTION}

Piperaceae grow in tropical forests from S Asia to Tropical America, including those of Africa and Australia. Althought TeBBS (1990) reduced Piperaceae to only 5 genera, most authors (YUNKER, 1958; DINIZ, 1997; IMMELMAN, 2000) include the 3000 species of the family in at least 7 genera.
Tropical Africa's Piperaceae were studied by BAKER \& WRIGHT (1913), VERDCOURT (1996), DINIZ (1997), and especially by HUTCHINSON \& DALZIEL $(1927-28,1954)$ and BALLE (1940-42; 1948) in West Tropical Africa. Further studies on Equatorial Guinea refer only to Bioko (ESCARRÉ, 1969; FernánDEZ CASAS 1992, 1995). There is no account of Piperaceae in Rio Muni, though they were 
included in Guinea's complete catalogue of vascular plants (GUINEA, 1946).

Over the last twenty years the Real Jardín Botánico, Madrid, has performed intensive fieldwork in both Bioko and Río Muni, in order to prepare a vascular Flora of Equatorial Guinea. First results with the identifications of these collections are those of VELAYOS \& al. (2001) or this check-list of Piperaceae.

The aim of this study is to update the catalogue of Piperaceae of the whole of Equatorial Guinea using both bibliographic records and herbarium material.

\section{MATERIAL AND METHODS}

This check-list is primarily based on herbarium specimens. We have studied the whole collection of Piperaceae of Equatorial Guinea from MA, including the collections of earlier Spanish expeditions (Guinea, Carvalho, Fernández Casas, etc.). In addition, we have included the collections made under the Agencia Española de Cooperacion Internacional project "Inventario florístico de Guinea Ecuatorial" -from 1998 to 2001- by Aedo, Pérez Viso and Velayos. These materials are deposited in MA. Bibliographic references on Piperaceae of Equatorial Guinea have been gathered too (AEDO \& al., 1999). We include one species based only on literature records, Peperomia pellucida (L.) Kunth, since its distribution area makes probably the presence of this species in Equatorial Guinea.

The locality names used in this check-list have been brought up-to-date and made uniform, according to VELAYOS \& al. (2001). For instance, we use Pico Basilé for "Clarence Peak", or "pico de Santa Isabel", etc.

The check-list is alphabetically ordered by genera and species. Generic and specific circumscription is in accordance with LEBRUN \& STORK (1991). Under each accepted name a list with the synonyms used in the literature of Equatorial Guinea and a bibliographic reference of a drawing of the species are included.

Herbarium specimens are ordered alphabetically by collectors within the different regions and provinces: Annobón, Bioko (provinces of Bioko Norte, and Bioko Sur) and Río Muni (provinces of Centro-Sur, NkieNtem, Litoral, Wele-Nzas). New records for each region are indicated under the species.

Previous bibliographical records of each taxon are grouped under the previously mentioned three regions. When the taxon was recorded under a different name (synonym or misidentification) it is indicated (i.e.: GUINEA, 1946: 271, sub $P$. mannii).

Pothomorphe, which taxonomic position is unclear, is treated as a separated genus, according to LEBRUN \& STORK (1991). Several authors prefer to reduce it to a section under Piper (TEBBS, 1990).

\section{CHECK-LisT}

\section{Peperomia fernandopoiana $C$. DC.}

Icon.: DüLL (1973: 80).

EQG, BIOKO NORTE: Malabo-Pico Basile, km 12, Canvalho 2951, MA 512080. Fernández Casas 10217. MA 511793. BIOKo SUR: Moka-Lago Loreto, Fernández Casas 11891. MA 513668. Belebú Balachá, Fernández Casas 12153, MA 513237. Alrededores del Lago Loreto. Guinea 1828, MA 387584.

Previously recorded from Annobón (DüL.L, 1973: 115), Bioko (DE CANDOLLE, 1866: 134; BAKER \& WRIGHT, 1909: 152; MILDBRAED, 1922: 181; HUTCHINSON \& DALZIEL, 1928: 79; 1954: 82; GUINEA, 1946: 271; 1968: 130; ESCARRÉ, 1969: 6-7; DÜLL, 1973: 114-115; JoHANSSON, 1974: 42; FERNÁNDEZ CASAS, 1992: 43) and Río Muni (GUINEA, 1946: 151).

\section{Peperomia kamerunana C. DC.}

Icon.: DüLL (1973: 76).

EQG, Bıoko Sur: Moka-Lago Biaó, Fernández Casas 10416, MA 512917.

Previously recorded from Bioko (HUTCHINSON \& DALZIEL, 1954: 83; ESCARRÉ, 1969: 5; DÜL, 1973: 95; FERNÁNDEZ CASAS, 1992: 43-44).

\section{Peperomia laeteviridis Engl.}

Icon.: DülL (1973: 78).

EQG, BIoko NoRTE: Malabo-Pico Basilé, Femández Casas 10318, MA 511668, Femández Casas 11228, MA 512661. 
Previously recorded from Bioko (DÜLL, 1973: 104; FERNÁNDEZ CASAS, 1992: 44; FERnÁndez Casas \& Morales, 1995: 238).

\section{Peperomia molleri C. DC.}

Icon.: DüLL (1973: 79).

EQG, CENTRo SuR: Bata-Niefang, Monte Alén, río Otom-Asok, Carvalho 5354, MA 598278.

Previously recorded from Bioko (HUTCHINSON \& DALZIEL, 1954: 82; ESCARRÉ, 1969: 6; DÜLL, 1973: 108; JOHANSSON, 1974: 42; Fernández Casas \& Morales, 1995: 238).

Not previously recorded from Río Muni.

\section{Peperomia pellucida (L.) Kunth}

Piper pellucidum L.

Peperomia vogelii Miq.

Icon.: DülL (1973: 73).

This species has been previosly recorded from Bioko: Bioko, unknown colector, (GUINEA, 1946: 271; CuFODONTIS, 1953: 3); Bioko Norte: Guinea 609 (HUTCHINSON \& DALZIEL, 1954: 82; ESCARRÉ, 1969: 6; DÜLL, 1973: 70; FERNÁNDEZ CASAS, 1992: 44), Escarré 395, 397 (ESCARRÉ, 1969: 6), Boughey 188 (DülL, 1973: 70; FERNÁNDEZ CASAS, 1992: 44) and Wrigley \& Melville 687 (FERNÁNDEZ CASAS, 1992: 44); Bioko Sur: Mildbraed 6805 (MILDBRAED, 1922: 181, sub Peperomia vogelii); Mildbraed 6803 (DüLL, 1973: 70, probably is the same collection that Millbraed recorded as Millbraed 6805), Fernández Casas 11424 (FERNÁNDEZ CASAS, 1992: 44).

We have not found any herbarium material of this species. Its distribution area suggests as acceptable the presence of this species in Equatorial Guinea (BALLE, 1940-42: 386; 1948: 22; ESCARRÉ, 1969: 6; VERDCOURT, 1996: 11; Diniz, 1997: 33; CABle \& ChEEK, 1998: 98).

\section{Peperomia retusa (L. f.) A. Dietr.}

Piper retusum L. f.

Peperomia retusa var. mannii (Hook. f) Düll Peperomia mannii Hook. $f$.

Peperomia buëana C. DC.
Icon.: DülL (1973: 75).

EQG, BIoko SUR: Moka-Ureka, Fernández Casas 11735, MA 513500. Moka-Lago Biaó, Fernández Casas 11951, MA 513588. Belebú Balachá-Ureka, Fernández Casas 12177, MA 512907.

Previously recorded from Bioko (HoOKER, 1864: 217, sub Peperomia mannii; BAKER \& WrIGHT, 1909: 153, sub P. mannii; MILDBRAED, 1922: 181a, sub $P$. mannii, 181b, sub $P$. buëana; Hutchinson \& DALZIEL, 1928: 80 , sub $P$. retusa var. mannii; 1954: 82 , sub $P$. mannii; GuINEA, 1946: 271, sub P. mannii; EsCARRÉ, 1969: 7, sub $P$. mannii; DülL, 1973: 91, sub P. retusa var. mannii; FERNÁNDEZ CASAS, 1992: 44, sub $P$. retusa var. mannii).

\section{Peperomia rotundifolia (L.) Kunth}

Piper rotundifolium $\mathrm{L}$.

Icon.: DüLL (1973: 74).

EQG, BIOKo NoRTE: Malabo-Pico Basile, km 3-4, Carvalho 4487, MA 511707.

Previously recorded from Bioko (FERNÁNdez Casas, 1992: 44; Fernández Casas \& MORALES, 1995: 238).

8. Peperomia tetraphylla (G. Forst.) Hook. \& Arn.

Piper tetraphyllum G. Forst.

Icon.: DÜLL (1973: 74).

EQG, Bıoko NorTE: Malabo-Pico Basilé, km 12, Carvalho 2642, MA 512233. Km 3-4, Carvalho 4524, MA 511682. Bioko SuR: Moka, Guinea 2277, MA 387562. Moka-Lago Loreto, Fernández Casas 11887, MA 513574; Lago Biá, Fernández Casas 11970, MA 513435 .

Previously recorded from Bioko (FERNÁNDEZ CASAS, 1992: 44; Fernández CaSAS \& MORALES, 1995: 238).

\section{Peperomia thomeana C. DC.}

Peperomia vaccinifolia C. DC.

Icon.: DüLL (1973: 80).

EQG, Bioko SuR: Lago Biaó, Fernández Casas 11966, MA 513443.

Previously recorded from Bioko (HUTCHINSON \& DALZIEL, 1954: 82, sub Peperomia 
vaccinifolia; ESCARRÉ, 1969: 7, sub $P$. vaccinifolia; DÜLL, 1973: 105; FERNÁNDEZ CASAS, 1992: 44).

\section{Peperomia vulcanica Baker \& C.H. Wright}

Peperomia hygrophila Engl.

Peperomia annobonensis Mildbr.

Icon.: DÜLL (1973: 80).

EQG, ANNOBON: Pico Surcado, Wrigley \& Melville 70, MA 294800. BıoKo NorTE: Malabo-Pico Basilé, km 5-6, Carvalho 4226, MA 514147. BIOKO SUR: Moka, fuentes de Mioco, Fernández Casas 11893, MA 513669.

Previously recorded from Annobón (MILDBRAED, 1922: 160, sub Peperomia annobonensis; EXELL, 1944: 276, sub P. annobonensis; GUINEA, 1946: 271, sub P. annobonensis; EXELL, 1963: 110, sub $P$. hygrophila; 1968: 134, sub P. hygrophila; DülL, 1973: 103) and Bioko (FERNÁNDEZ CASAS, 1992: 44-45; FERNÁNDEZ CASAS \& MoRALES, 1995: 238).

\section{Piper capense L. f.}

\section{Coccobryon capense (L. f.) Klotzsch}

Icon.: ENGLER (1910: 649); BALLE (1948: 19).

EQG, BIoKo NoRTE: Malabo-Pico Basilé, Carvalho 2368, MA 512226; Fernández Casas 10169, MA 511711; Fernández Casas 11233, MA 512666. Bıoko SUR: Moka-Riaba, camino viejo, Fernández Casas 11808, MA 513252. Moka-Lago Biao, Fernández Casas 11942, MA 513595. Musola, servicio agronómico, Guinea 1194, MA 412217. Musola, Finca Puente, Guinea 1752, MA 294858; Guinea 1753, MA 412216. CENTro SuR: Bata-Monte Alén, rio Otom-Asok, Carvalho 5331, MA 598178.

Previously recorded from Bioko (HOOKER, 1864: 217, sub Coccobryon capense; BAKER \& Wright, 1909: 146; MildBRAED, 1922: 181; HUTCHINSON \& DALZIEL, 1928: 80; 1954: 84; GUINEA, 1946: 271; CUFODONTIS, 1953: 3; ESCARRÉ, 1969: 8; FERNÁNDEZ CASAS, 1992: 45).

Not previously recorded from Río Muni.

12. Piper guineense Schum. \& Thonn.

Piper clusii C. DC.

Icon.: ENGLER (1910: 649); BALLE (1940-42: 379).
Common names. Spanish: pimienta de Guinea (GuINEA, 1946). Bubi dialect: Topotópoto, Tökolo to muan.

EQG, BIoko NoRTE: Malabo-Pico Basilé, km 3, Carvalho 2269, MA 512203. Malabo-Cupapa, km 18-19, Carvalho 3476. MA 511908; km 22-23, Carvalho 4131, MA 655094. Malabo-Basilé, Finca Vigatana, Fernández Casas, Carvalho, Regueiro \& Tellería 10103, MA 399760. 407526. Malabo-Riaba, Bariobé, Fernández Casas 10219, MA 511794. Cupapa, Fernández Casas 11494, MA 512919; Malabo-Punta Hermosa, km 11-12, Fernández Casas 12090, MA 513360. Misión Católica de Malabo, Guinea 778, MA 412218; Guinea 780, MA 294879. BıKo SuR: Ruiché, Fernández Casas 11346, MA 512790. LubaMalabo, km 40, Fernández Casas 12215, MA 512647. CENTRO SUR: Bata-Niefang, km 35, Mboete, Carvalho 5548 , MA 598581; Carvalho 5590, MA 598813. P. N. Monte Alén, Pérez Viso 95, MA 655093.

Previously recorded from Bioko (BAKER \& WRIGHT, 1909: 145; MILDBRAED, 1922: 181; HUTCHINSON \& DALZIEL, 1928: 80; 1954: 84; GuINEA, 1946: 271a; 271b, sub Piper clusii; 1968: 130; EsCARRÉ, 1969: 9; FERNÁNDEZ CASAS, 1992: 45a; 45b, sub Piper capense L. f.) and Río Muni (GuINEA, 1946: 142, 149, $151,155)$.

\section{Pothomorphe umbellata (L.) Miq.}

Piper umbellatum $\mathrm{L}$.

Piper subpeltatum Willd.

Heckeria subpeltata (Willd.) Kunth

Icon.: HUTCHINSON \& DALZIEL (1954: 83); DINIZ (1997: 26).

Common names. Fang dialect: Abomenjan, Abomenchan, Abomndzan (GuINEA, 1946). Bubi dialect: Lootáotá, Mëvotö.

EQG, ANNobón: S Lago A Pot. Wrigley \& Melville 147, MA 294974. BIOKO NORTE: Malabo-Rebola, km 1011, Fernández Casas 11262, MA 512639. MalaboRiaba, Baloeri, Carvalho 2493, MA 512114. Baó Basuala, Fernández Casas 11506, MA 513519. Monte Balea, Guinea 366, MA 412215. BIOKO SUR: Basacato del Oeste, Fernández Casas, Carvalho, Regueiro \& Tellería 10060, MA 425807. Malabo-Luba, km 32, Fernández Casas 11318, MA 512583. Ruiche-Luba, Fernandez Casas 11357, MA 512783. Basacato del Oeste, Lope del Val 469, MA 389402. Playa de Arenas Blancas, Fernández Casas 11652, MA 513750. Luba-Veiga y Avendaño, límite Batete-Luba, Fernández Casas 12024, MA 513362. Moka-Riaba, km 7-8, Fernández Casas 11601, MA 513218. Belebú Balachá, Fernández Casas 12127, MA 513229. Centro Sur: P. N. Monte Alén. Pérez Viso 54, MA 655092. P. N. Monte Alén, Ocuam, Pérez Viso 618, MA 655090. P. N. Monte Alén, Miser- 
gue-Churu, Pérez Viso 1641, MA 655089. Bata-Niefang, Nhogo Ebaiñ, Pérez Viso 2671 bis, MA 655088. NiefangEvinayong, Bisun, Pérez Viso 3427, MA 655087. Bindung, Pérez Viso 3781, MA 655086. Litoral: BataKogo, km 67, Pérez Viso 1846, MA 655091.

Previously recorded from Annobón (MiLDBRAED, 1922: 160, sub Heckeria subpeltata; EXELL, 1944: 276, sub Piper umbellatum), Bioko (BAKER \& WRIGHT, 1909: 145, sub P. umbellatum; MILDBRAED, 1922: 181, sub $P$. subpeltatum; GuINEA, 1946: 271, sub $P$. subpeltatum; 1968: 130, sub $P$. umbellatum; HUTCHINSON \& DALZIEL, 1954: 84, sub P. umbellatum; ESCARRÉ, 1969: 8, sub P. umbellatum; FERNÁNDEZ CASAS, 1992: 45, sub P. umbellatum) and Río Muni (GUINEA, 1946: 142, sub Heckeria subpeltata, 151, sub $H$. subpeltata, 157 , sub $H$. subpeltata; 1946 : 271, sub $P$. subpeltatum).

\section{REFERENCES}

Aedo, C., M.T. Tellería \& M. Velayos (1999). Bases Documentales para la Flora de Guinea Ecuatorial. Plantas vasculares y hongos. Real Jardín Botánico (CSIC). Cooperaciớn Española. Madrid.

Balle, S. (1940-1942). Revision des Piperaceae du Congo Belge. Bull. Jard. Bot. État 16: 367-405.

BaLLE, S. (1948). Piperaceae. In: W. Robyns (ed.), Flore du Congo Belge et du Ruanda-Urundi. Spermatophytes 1: 15-27. Bruxelles.

BAKER, J.G. \& C.H. WRIGHT (1909-1913). Piperaceae. In: W.T. Thiselton-Dyer (ed.), Flora of Tropical Africa 6(1): 143-156. Lovell Reeve \& Co., London.

Cable, S. \& M. Cheek (1998). The Plants of Mount Cameroon. A Conservation Checklist. Royal Botanic Gardens, Kew.

CuFodonts, G. (1953). Enumeratio Plantarum Aethiopiae Spermatophyta. Bull. Jard. Bot. Etat 23(3-4) Suppl.: i-xvi, 1-112.

De Candolle, C. (1866). Piperaceae novae. J. Bot., ser. 2, 4: 132-146.

DinIz, M.A. (1997). Piperaceae. In: G.V. Pope, S.J. Owens \& I. Moreira (eds.). Flora Zambesiaca 9(2): 24-36. Royal Botanic Garden, Kew for the Flora Zambesiaca Managing Committee.

Düll, R. (1973). Die Peperomia-Arten Afrikas. Bot. Jahrb. Syst. 93: 56-129.

ENGLER, A. \& O. DRUDE (1910). Die Vegetation der Erde. Die Pflanzenwelt Afrikas insbesondere seiner tropischen Gebiete. Leipzig.

ESCARRÉ, A. (1969). Aportaciones al conocimiento de la Flora de Fernando Poo. 2. Piperaceae, Urticaceae. Acta Phytotax. Barcinon. 3: 1-23.

EXELL, A.W. (1944). Catalogue of the vascular plants of S. Tome (with Principe and Annobon). The British Museum (Natural History). London.
ExEL, A.W. (1963). Angiosperms of the Cambridge Annobon Island Expedition. Bull. Brit. Mus. (Nat. Hist.), Bot. 3(3): 93-118.

Exell, A.W. (1968). Príncipe, S. Tomé \& Annobón. In: 1. Hedberg \& O. Hedberg (eds.), Conservation of vegetation in Africa South of the Sahara. Acta Phytogeogr. Suec. 54(11): 132-134.

FERNÁNDEZ CASAS, F.J. (1992). Ad Guineae Aequatorialis floram texendam inventa varia. Fontqueria 33: 43-45.

Fernández Casas, F.J. \& R. Morales Valverde (1995). Proyecto de una flora de la isla de Bioko (Guinea Ecuatorial). Anales Jard. Bot. Madrid 52(2): 230-240.

GUINEA, E. (1946). Ensayo geobotánico de la Guinea Continental Española. Dirección General de Marruecos y Colonias. Madrid.

Guinea, E. (1968). Fernando Po. In: I. Hedberg \& $O$. Hedberg (eds.), Conservation of vegetation in Africa South of the Sahara. Acta Phytogeogr. Suec. 54: $x i, 130-132$.

HoOKER, J.D. (1864). On the plants of the temperate regions of the Cameroons Mountains and Islands in the Bight of Benin; collected by Mr. Gustav Mann, Government Botanist. J. Proc. Linn. Soc., Bot. 7: 171240.

Hutchinson, J. \& J.M. DALZIEL (1927-1928). Flora of West Tropical Africa. 1(1). The Crown Agents for the Colonies.

Hutchinson, J. \& I.M. DalzJEL (1954). Flora of West Tropical Africa. 2nd. ed. 1(1). The Crown Agents for Oversea Governments and Administrations.

ImmelmaN, K.L. (2000). FSA contributions 15: Piperaceae. Bothalia 30(1); 25-30.

JOHANSSON, D. (1974). Ecology of vascular epiphytes in West African rain forest. Acta Phytogeogr. Suec. 59: 1-129.

LeBRUN, J.P. \& L.A. STORK (1991). Énumération des Plantes à Fleures D'Afrique Tropicale. I. Généralités et Annonaceae à Euphorbiaceae et Pandaceae. Conservatoire et Jardin Botaniques de Genève.

MILDBRAED, G.W.J. (1922). Wissenschaftliche Ergebnisse der Zweiten Deutschen Zentral-Afrika-Expedition 1910-1911. Band II: Botanik. Verlag von Klinkhardt \& Biermann. Leipzig.

Tefess, M.C. (1990). Piperaceae. In: K. Kubitzki, J.G. Rohwer \& V. Bittrich (eds.), The Families and Genera of Vascular Plants. II. Flowering Plants, Dicotyledons. Magnoliid, Hamamelid and Caryophyllid Families. Springer-Verlag, Berlin.

Velayos, M., C. Aedo \& R. Pérez Viso (2001). CheckList of The Pteridophytes of Equatorial Guinea. Belg. J. Bot. 134(2): 145-191.

Verdcourt, B. (1996). Piperaceae. In: R.M. Polhill (ed.), Flora of Tropical East Africa: 1-23. A.A. Balkema, Rotterdam.

YUNKER, T.G. (1958). The Piperaceae-A Family Profile. Brittonia 10(1): 1-7.

YUNKER, T.G. (1964). A bibliography of the family Piperaceae. Candollea 19: 97-144. 


\section{COLLECTION INDEX}

The species is indicated by a number in parenthesis corresponding to the number in the check-list.

Carvalho 2493: (13); 2642: (8); 2951: (1); 3476: (12); 4131:(12); 4226: (10); 4487: (7); 4524: (8); 5331: (11); 5354: (4); 5548: (12); 5590: (12).

Escarré 395: (5); 397: (5).

Fernández Casas 10060: (13); 10103: (12); 10169: (11); 10217: (1); 10219: (12); 10318: (3); 10416: (2); 11228: (3); 11233: (11); 11262: (13); 11318: (13); 11346: (12); 11357: (13); 11424: (5); 11494: (12); 11506: (13); 11601: (13); 11652: (13); 11735: (6); 11808: (11); 11887: (8); 11891: (1); 11893: (10); 11942: (11); 11966: (9); 11951: (6); 11970: (8); 12024: (13); 12090: (12); 12127: (13); 12153: (1); 12177: (6); 12215: (12).

Guinea 366: (13); 609: (5); 778: (12); 780: (12); 1194: (11); 1752 (11); 1753: (11); 1828: (1); 2277: (8).

Lope del Val 469: (13).

Mildbraed 6805: (5).

Pérez Viso 54: (13); 95: (12); 618: (13); 641: (13); 1846:

(13); 2671 bis: (13); 3427: (13); 3781: (13).

Wrigley \& Melville 70: (10); 147: (13); 687: (5).
Peperomia annobonensis Mildbr., 48 Peperomia buëana C. DC., 47

Peperomia fernandopoiana C. DC., 46

Peperomia hygrophila Engl., 48

Peperomia kamerunana C. DC., 46

Peperomia laeteviridis Engl., 46

Peperomia mannii Hook. f., 47

Peperomia molleri C. DC., 47

Peperomis pellucida (L.) Kunth. 47

Peperomia retusa (L. f.) A. Dietr., 47

Peperomia retusa var, mannii (Hook. f) Düll, 47

Peperomia rotundifolia (L.) Kunth, 47

Peperomia tetraphylla (G. Forst.) Hook. \& Arn., 47

Peperomia thomeana C. DC., 47

Peperomia vaccinifolia C. DC., 47

Peperomia vogelli Miq., 47

Peperomia vulcanica Baker \& C.H. Wright, 48

Piper capense L. f., 48

Piper guineense Schum. \& Thonn., 48

Piper pellucidum L., 47

Piper retusum L. f., 47

Piper rotundifolium L., 47

Piper subpeltatum Willd., 48

Piper tetraphyllum G. Forst., 47

Piper umbellatum L., 48

Pothomorphe umbellata (L.) Miq., 48

\section{INDEX TO SCIENTIFIC NAMES}

Coccobryon capense (L. f.) Klotzsch, 48

Heckeria subpeltata (Willd.) Kunth, 48

Editado por Juan José Aldasoro Aceptado para publicación: 29-XI-2002 